\title{
A push forward construction and the comprehensive factorization for internal crossed modules
}

\author{
Dedicated to George Janelidze on the occasion of his 60th birthday
}

\author{
Alan S. Cigoli, Sandra Mantovani, Giuseppe Metere
}

November 15, 2012

\begin{abstract}
In a semi-abelian category, we give a categorical construction of the push forward of an internal pre-crossed module, generalizing the pushout of a short exact sequence in abelian categories. The main properties of the push forward are discussed. A simplified version is given for action accessible categories, providing examples in the categories of rings and Lie algebras. We show that push forwards can be used to obtain the crossed module version of the comprehensive factorization for internal groupoids.
\end{abstract}

\section{Introduction}

Given a short exact sequence $0 \rightarrow A \rightarrow X \rightarrow Y \rightarrow 0$ in an abelian category $\mathcal{A}$, any morphism $y: Y^{\prime} \rightarrow Y$ produces by pullback a new short exact sequence with the same kernel $A$. Dually, any morphism $a: A \rightarrow A^{\prime}$ produces by pushout a new short exact sequence with the same cokernel $Y$. These constructions are functorial (the functors thus obtained are denoted by $y^{*}$ and $a_{*}$ ) and they give rise to the classical homomorphisms:

$$
\begin{gathered}
\operatorname{Ext}_{\mathcal{A}}(y, A): \operatorname{Ext}_{\mathcal{A}}\left(Y^{\prime}, A^{\prime}\right) \rightarrow \operatorname{Ext}_{\mathcal{A}}\left(Y, A^{\prime}\right) \\
\operatorname{Ext}_{\mathcal{A}}(Y, a): \operatorname{Ext}_{\mathcal{A}}(Y, A) \rightarrow \operatorname{Ext}_{\mathcal{A}}\left(Y, A^{\prime}\right)
\end{gathered}
$$

If the base category is a semi-abelian category $\mathcal{C}$, the first construction still produces a short exact sequence, but this is no longer true for the second one, because the pushout of a normal monomorphism is not in general a normal monomorphism.

This problem can be fixed by giving some supplementary conditions, and a push forward construction, that in the abelian case specializes to a pushout.

For extensions with abelian kernel, this issue is discussed in literature, especially in connection with the different interpretations of low dimensional non abelian cohomological theories (see e.g. [15], Chapter IV, or [10], Chapter IV). 
Indeed, for extensions with abelian kernel, the push forward construction makes the following classical assignment functorial:

$$
\left.\operatorname{Opext}_{\mathcal{C}}(Y,-) \text { : [Abelian objects in } \mathcal{C} \downarrow Y\right] \rightarrow[\text { Abelian groups }]
$$

(see [1] for groups and other algebraic categories, and [7] for pointed protomodular categories). Here, the supplementary conditions ensure that $a$ gives rise to a morphism of abelian group objects in $\mathcal{C} \downarrow Y$. Indeed, the same situation can be treated also with the alternative approach developed by Bourn in [4], where a direction functor assigns to each abelian extension of the object $Y$ a $Y$-module. Under this perspective, the push forward construction recovers the fact that such direction functor is a pseudo cofibration (see [4]).

A generalization of the push forward construction arises when we consider the normal monomorphism appearing in any short exact sequence as an instance of a pre-crossed module.

When the base category is the category of groups, under suitable hypothesis, it is possible to push forward along a map not only a normal monomorphism, but any pre-crossed module. This way we obtain a crossed module with the same cokernel (to the best of our knowledge, the push forward of a pre-crossed module was introduced by Noohi in [20]).

The purpose of the present work is to develop a push forward construction in the intrinsic setting of a semi-abelian category $\mathcal{C}$, where the notion of internal crossed module was introduced by Janelidze in [12].

In fact, in Theorem 3.6, we present necessary and sufficient conditions, expressed in terms of internal actions, for the push forward of a given pre-crossed module to exist in $\mathcal{C}$ (for the case of a crossed module, a push forward construction with different conditions was independently developed by Hartl [11]). These conditions simplify when the category $\mathcal{C}$ is action accessible [8], as presented in Theorem 3.10. The last result is very useful for the construction of push forward in many algebraic examples, like those of rings, Lie algebras, associative algebras and, more in general, any category of interest in the sense of Orzech [21].

The push forward construction, together with its main property (see Theorem 3.6, $(\mathrm{PF})$ ), turns out to be strongly related to the comprehensive factorization of internal functors (see [3]). Our investigation shows that push forwards can be used in order to factorize morphisms of crossed modules, so that final functors between internal groupoids can be characterized as push forward squares.

\section{Contents}

\section{Introduction}

2 Preliminaries 3

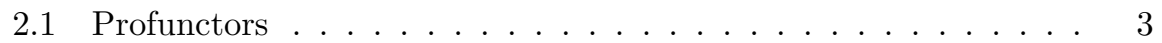

2.2 Double groupoids in discrete fibrations . . . . . . . . . . . 5 
3 Push forward of pre-crossed modules 6

3.1 The main result . . . . . . . . . . . . . . . 6

3.2 The case of action accessible categories . . . . . . . . . 15

3.3 A useful construction for the push forward of pre-crossed modules 18

3.4 A characterization . . . . . . . . . . . . . . . . 20

4 Examples 22

4.1 Rings . . . . . . . . . . . . . . . . . . . . 23

4.2 Lie and Leibniz algebras . . . . . . . . . . . . . . . . 24

5 Push forward of extensions $\quad 25$

5.1 Abelian extensions . . . . . . . . . . . . . . . . . 25

5.2 General case . . . . . . . . . . . . . . . . . . . . 28

6 Push forward and the comprehensive factorization for internal $\begin{array}{ll}\text { crossed modules } & 29\end{array}$

6.1 Factorization systems for internal crossed modules . . . . . . . . 29

6.2 Final morphisms of internal crossed modules . . . . . . . . 30

6.3 Factorization of morphisms of extensions . . . . . . . . . 33

7 Acknowledgments

\section{Preliminaries}

In the sequel we will use the notion of profunctor between groupoids.

\subsection{Profunctors}

Let $\mathcal{C}$ be a category with finite limits. A morphism $\underline{f}=\left(f_{1}, f_{0}\right): \underline{E} \rightarrow \underline{H}$ of reflexive graphs in $\mathcal{C}$ :

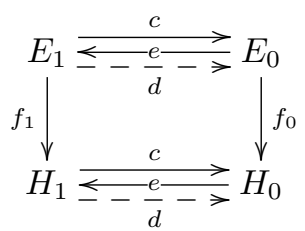

is a discrete fibration if the square

$$
c \cdot f_{1}=f_{0} \cdot c
$$

is a pullback. In the case where $f$ is a functor between internal categories, this is equivalent to saying that the domain map $d: E_{1} \rightarrow E_{0}$ gives a right action of $\underline{H}$ on $E_{0}$. The internal dual of this definition is the notion of discrete cofibration, that is, a morphism $f: \underline{E} \rightarrow \underline{H}$ for which the square

$$
d \cdot f_{1}=f_{0} \cdot d
$$


is a pullback. This two notions are different in general, while in the case of functors between groupoids they imply each other.

We denote by $\operatorname{Gpd}(\mathcal{C})$ the category of internal groupoids and internal functors in $\mathcal{C}$ and by $\operatorname{Gpd}_{d f}(\mathcal{C})$ the category of internal groupoids in $\mathcal{C}$ with discrete fibrations as morphisms.

In the set-theoretical context, a profunctor between categories $\underline{H} \rightarrow \underline{G}$ is a functor $\underline{H} \times \underline{G}^{o p} \rightarrow$ Set. Alternatively, a profunctor can be described in terms of a discrete fibration over $\underline{H}$ and a discrete cofibration over $\underline{G}$. This description is internal, hence it yields a definition of internal profunctor (see [22]).

Definition 2.1. Let $\mathcal{C}$ be a finitely complete category. A profunctor $E: \underline{H} \rightarrow \underline{G}$ is given by a discrete fibration on $\underline{H}$ and a discrete cofibration on $\underline{G}$ as in the diagram

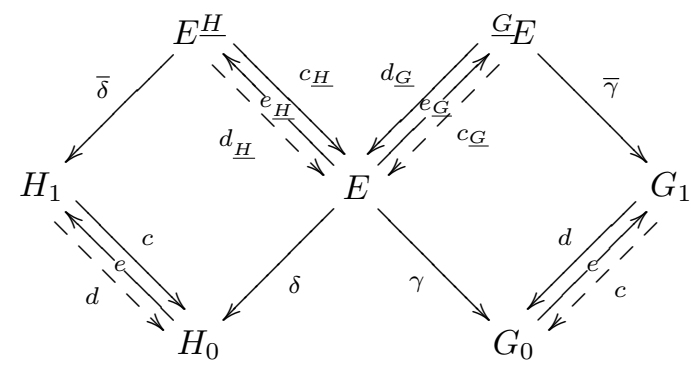

such that the two actions on $E$ are compatible and commute (see [18]).

Recall from [22] that any internal functor $\underline{f}: \underline{H} \rightarrow \underline{G}$ between internal categories in $\mathcal{C}$ gives rise to a profunctor $\underline{f}: \underline{H} \rightarrow \underline{G}$ as follows: given the functor

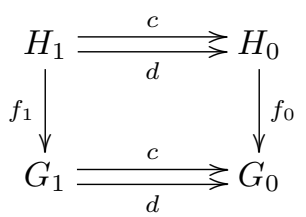

consider the pullback

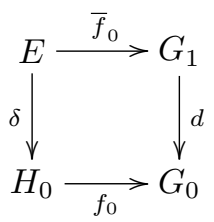


and define the profunctor $\underset{f}{\text {. by }}$

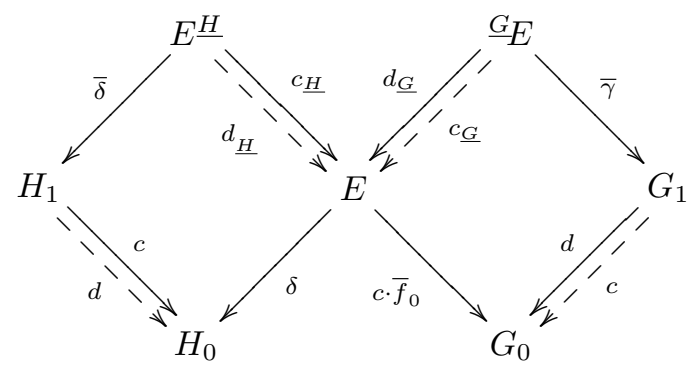

When the categories involved are groupoids, discrete fibrations are also discrete cofibrations, and vice versa. As a consequence, the notion of profunctor symmetrizes, and the groupoids $\underline{E}^{\underline{H}}$ and $\underline{\underline{G}}$ are connected ([5]), i.e. the two groupoids give rise to a double groupoid in discrete fibrations:

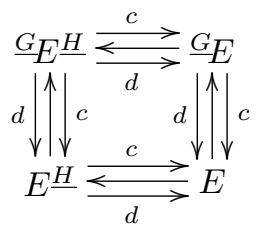

Actually, all the paired squares in the diagram above are pullbacks. This yields a double centralizing groupoid of $\underline{E}^{\underline{H}}$ and $\underline{\underline{E}} \underline{E}$ in the sense of Bourn. When the base category is Barr-exact and Malt'sev, this is unique, and it is called the core of the profunctor $E: \underline{H} \rightarrow \underline{G}$ (see [5]).

\subsection{Double groupoids in discrete fibrations}

Let us recall the following basic facts and definitions. Let $\mathcal{C}$ be a category with finite limits. A double groupoid in $\mathcal{C}$ is an object of $\operatorname{Gpd}(\operatorname{Gpd}(\mathcal{C}))$. It is a general fact that a double groupoid, likewise any double category, can be interpreted as having as objects either the vertical arrows or the horizontal arrows.

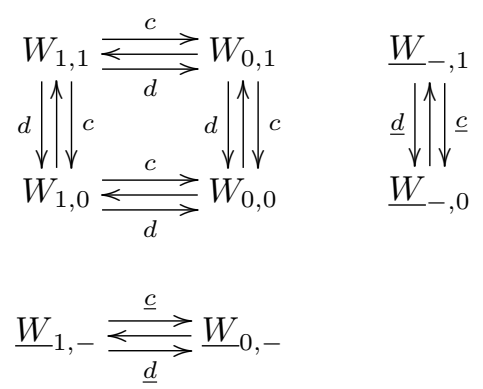

In the above picture, the upper square diagram on the left hand side is the double groupoid $\underline{\underline{W}}$, with horizontal arrows $W_{1,0}$ and vertical arrows $W_{0,1}$. The 
diagram on the right hand side describes it as a groupoid with objects vertical arrows, while the diagram on the bottom as a groupoid with objects horizontal arrows.

We say that a double groupoid is in discrete fibrations when it is an object of $\operatorname{Gpd}\left(\operatorname{Gpd}_{d f}(\mathcal{C})\right)$.

We recall from [9] the following very important property which holds in regular Mal'tsev categories.

Proposition 2.2 ([9], 3.4). Let $\mathcal{C}$ be a regular Mal'tsev category. Suppose that the following whole rectangle is a pullback and the left hand side is a commutative square of vertical split epimorphisms:

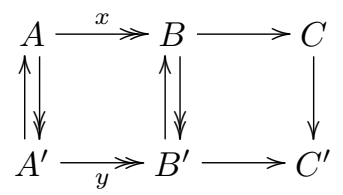

If $x$ (and thus $y$ ) is a regular epimorphism, then the two squares are pullbacks.

Corollary 2.3. Let $\mathcal{C}$ be a regular Mal'tsev category. Consider a split epimorphic discrete fibration and cofibration of internal reflexive graphs in $\mathcal{C}$, i.e. a split epimorphism $\underline{G} \rightarrow \underline{H}$ of reflexive graphs

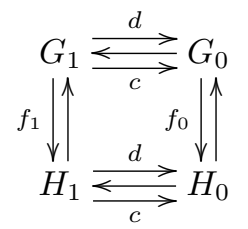

where both the squares $d f_{1}=f_{0} d$ and $c f_{1}=f_{0} c$ are pullbacks.

Let $R$ and $S$ be the supports of $\underline{G}$ and $\underline{H}$ respectively ( $R$ and $S$ are equivalence relations since they are reflexive relations and $\mathcal{C}$ is a Mal'tsev category).

Then the diagram above factorizes through a discrete fibration between $R$ and $S$.

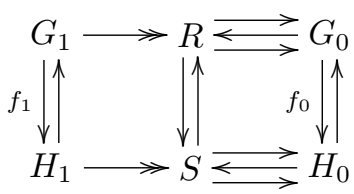

Proof. Trivial by Proposition 2.2.

\section{Push forward of pre-crossed modules}

\subsection{The main result}

In the following, the base category $\mathcal{C}$ is supposed to be semi-abelian. For a detailed account on semi-abelian categories, semi-direct products and internal actions, the reader is adressed to [2] and [6]. We just recall that in the semi-abelian 
case there is a correspondence between internal actions and split epimorphisms with a chosen section.

Lemma 3.1. Let $p: A \rightarrow B$ be a split epimorphism, with chosen section $s$, and $f: E \rightarrow B$ be a morphism. Consider the following pullback diagram of split epimorphisms

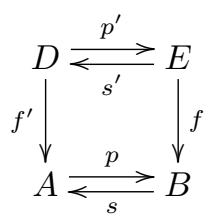

If $\xi: B b X \rightarrow X$ is the internal action determined by $(p, s)$ on its kernel $X$, then $f^{*}(\xi)=\xi \cdot\left(f b 1_{X}\right)$ is the internal action determined by $\left(p^{\prime}, s^{\prime}\right)$.

Proof. Trivial.

Definition 3.2. A pre-crossed module $\left(\partial_{H}, \xi_{H}\right)$ is a morphism $\partial_{H}: H \rightarrow H_{0}$ together with an action $\xi_{H}: H_{0} b H \rightarrow H$ such that the following diagram commutes:

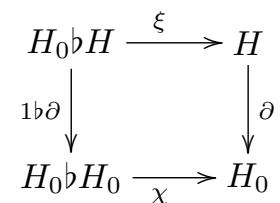

where $\chi=\chi_{H_{0}}$ is the canonical conjugation action.

Subscripts are omitted when no confusion arises.

Pre-crossed modules cerrespond to reflexive graphs (see [12]). The construction is based on the semidirect product $H \rtimes_{\xi} H_{0}$ : the codomain map is the canonical projection $p_{H_{0}}$, while the domain is the unique arrow $\left[\partial, 1_{H_{0}}\right\rangle$ that makes the two triangles commute:

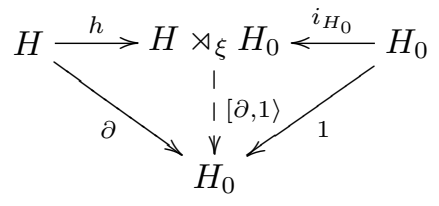

In order to define an internal crossed module, we need a further condition, which is not in general the straightforward generalization of the Peiffer condition for crossed modules of groups (see e.g. [16]). In fact, Janelidze in [12] gave a definition of internal crossed module, showing the equivalence between the category of crossed modules and the one of internal groupoids. 
Definition $3.3([12])$. A crossed module is a pre-crossed module $(\partial, \xi)$ such that the following diagram commutes:

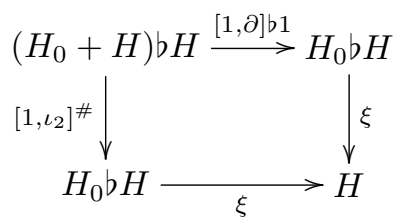

where the arrow $\left[1, \iota_{2}\right]^{\#}$ is defined by the following diagram:

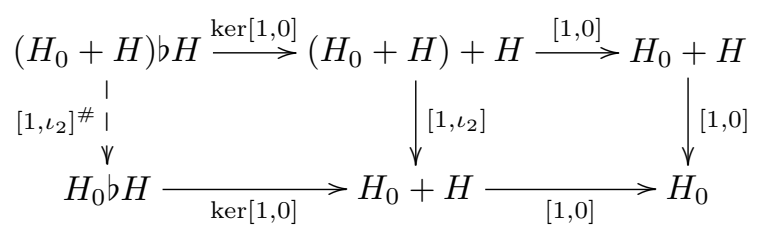

The following proposition gives an alternative definition of crossed module.

Proposition 3.4. Let $(\partial, \xi)$ be a pre-crossed module. Then the following conditions are equivalent:

1. $(\partial, \xi)$ is a crossed module;

2. the following diagram commutes:

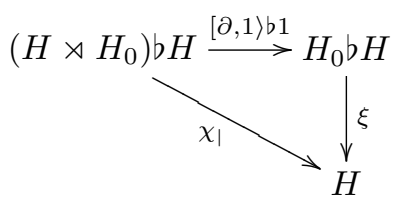

where the map $\chi_{\mid}=\chi_{H}^{H \rtimes H_{0}}$ is the conjugation action of $H \rtimes H_{0}$ restricted to the inclusion $i_{H}: H \rightarrow H \rtimes H_{0}$.

Proof. Let us consider the following commutative diagrams:

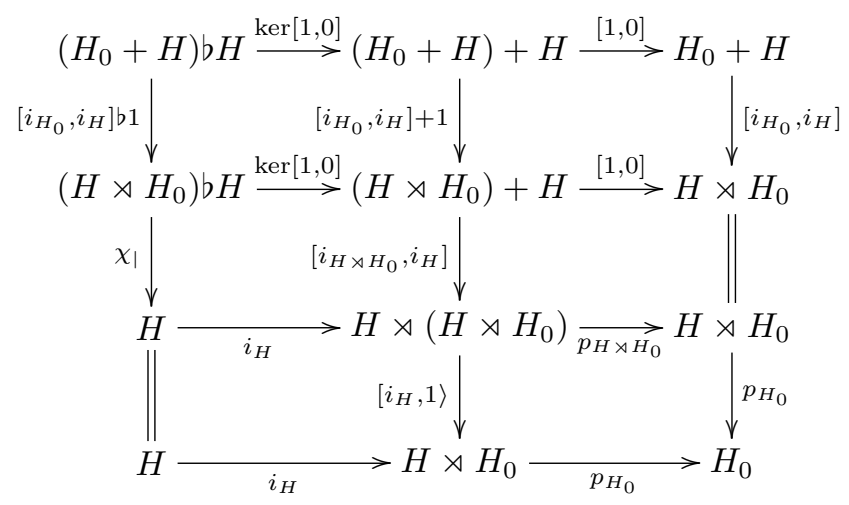




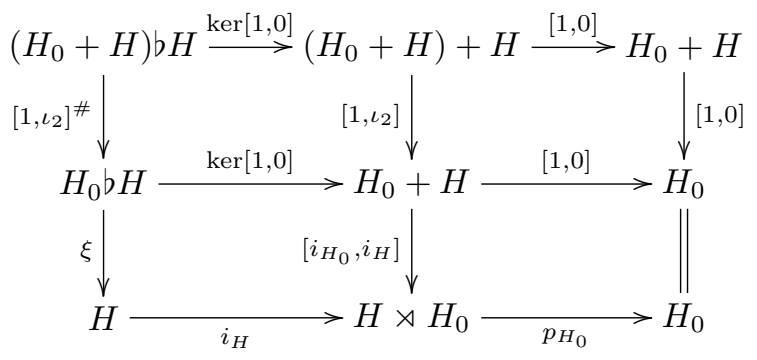

We have that

$$
\xi \cdot\left[1, \iota_{2}\right]^{\#}=\chi_{\mid} \cdot\left[i_{H_{0}}, i_{H}\right] b 1,
$$

since they are the restriction to kernels of the same morphism between extensions. Now, if Condition 2 holds, then

$$
\xi \cdot\left[1, \iota_{2}\right]^{\#}=\xi \cdot[\partial, 1\rangle b 1 \cdot\left[i_{H_{0}}, i_{H}\right] b 1=\xi \cdot[\partial, 1] b 1,
$$

i.e. $(\partial, \xi)$ is a crossed module. The converse implication follows from the fact that $\left[i_{H_{0}}, i_{H}\right]$ is a regular epimorphism and $-b 1$ preserves such morphisms (see $[12])$.

Remark 3.5. If we pre-compose diagram 2 of the above proposition with the morphism

$$
i_{H} b 1: H b H \rightarrow\left(H \rtimes H_{0}\right) b H
$$

we obtain the Peiffer condition (see $[12,16])$ :

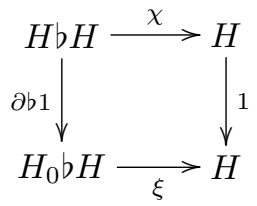

which is weaker, in general, than the above equivalent conditions. However, if the category $\mathcal{C}$ satisfies the "Smith is Huq" property, the Peiffer condition turns out to be sufficient to characterize internal crossed modules among pre-crossed modules (see [19]).

Now we are ready to prove our main result on the push forward construction.

Theorem 3.6. In a semi-abelian category, we consider a pre-crossed module $\left(\partial: H \rightarrow H_{0}, \xi\right)$, a morphism $p: H \rightarrow G$, and an internal action $\alpha: H_{0} b G \rightarrow G$ such that the following diagram commutes:

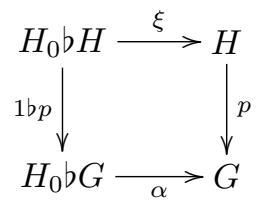


or, equivalently, the arrow $(p \rtimes 1): H \rtimes_{\xi} H_{0} \rightarrow G \rtimes_{\alpha} H_{0}$ exists. Moreover we require that the following diagram commutes:

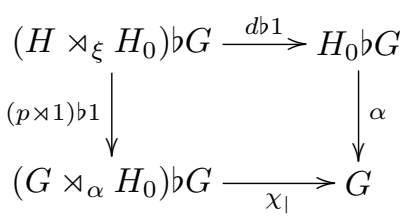

where $d=[\partial, 1\rangle: H \rtimes_{\xi} H_{0} \rightarrow H_{0}$ is the domain map determined by the precrossed module $(\partial, \xi)$, and $\chi_{\mid}=\chi_{G}^{G \rtimes H_{0}}$.

Then there exist:

1. an object $G \rtimes^{H} H_{0}$;

2. a crossed module $\left(\widetilde{\partial}: G \rightarrow G \rtimes^{H} H_{0}, \widetilde{\xi}\right)$, with $\operatorname{coker}(\widetilde{\partial}) \cong \operatorname{coker}(\partial)$;

3. a morphism $\widetilde{p_{0}}: H_{0} \rightarrow G \rtimes^{H} H_{0}$, such that $\left(p, \widetilde{p_{0}}\right)$ is a morphism of precrossed modules.

This construction is characterized by the following property:

$(\mathrm{PF})$ for any morphism $\left(p, p_{0}\right)$ from $(\partial, \xi)$ to a crossed module $\left(\partial^{\prime}: G \rightarrow G_{0}, \xi^{\prime}\right)$, with $p_{0}^{*}\left(\xi^{\prime}\right)=\alpha$, there exists a unique arrow $t: G \rtimes^{H} H_{0} \rightarrow G_{0}$ such that $t \widetilde{p_{0}}=p_{0}$ and $\left(1_{G}, t\right)$ is a morphism of crossed modules:

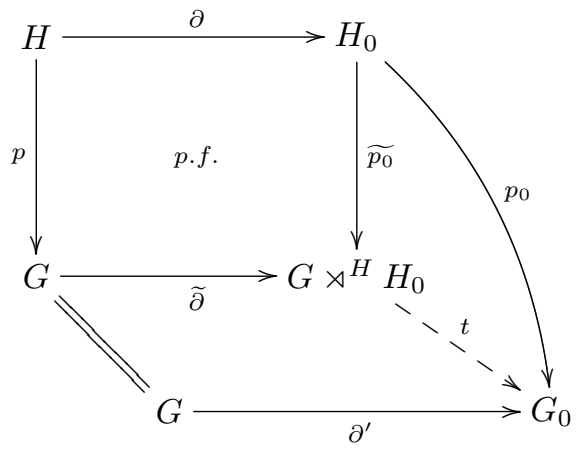

The object $G \rtimes^{H} H_{0}$ is called generalized semi-direct product, and the crossed module $\widetilde{\partial}$ is called the push forward of $\partial$ along $p$.

Proof. Consider the following pullback of split epimorphisms:

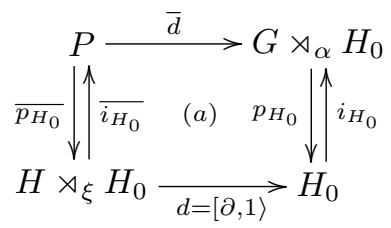


By Lemma 3.1, the hypothesis given by the commutative diagram (3) says that in the pullback $(b)$ below we can choose the same point $\left(\overline{p_{H_{0}}}, \overline{i_{H_{0}}}\right)$ as before:

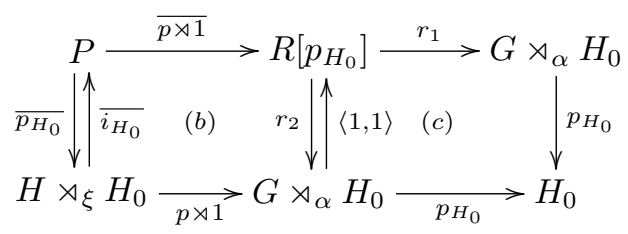

The downward pullbacks $(b)$ and $(c)$ paste together, so that $(a)$ with $(b)+(c)$ give a discrete fibration and cofibration of reflexive graphs:

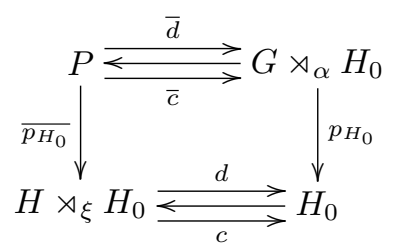

where the codomain map $c: H \rtimes_{\xi} H_{0} \rightarrow H_{0}$ is indeed the projection onto $H_{0}$, i.e. the composition $p_{H_{0}} \cdot(p \rtimes 1)$ in diagram (4) above, so that $\bar{c}=r_{1} \cdot \overline{p \rtimes 1}$.

Now consider the kernel pairs of $\overline{p_{H_{0}}}$ and $p_{H_{0}}$, with the induced morphisms between them:

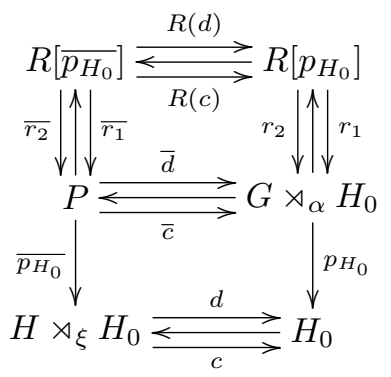

As the lower commutative squares are pullbacks, the four commutative squares at the top are also pullbacks. Since the base category is semi-abelian, it is Mal'tsev and regular, hence by Corollary 2.3 the split discrete fibrations/cofibrations $\left(\overline{r_{1}}, r_{1}\right)$ and $\left(\overline{r_{2}}, r_{2}\right)$ between the reflexive graphs $\left(P, G \rtimes_{\alpha} H_{0}\right)$ and $\left(R\left[\overline{p_{H_{0}}}\right], R\left[p_{H_{0}}\right]\right)$ factorize through discrete fibrations between their supports $R$ and $S$ :

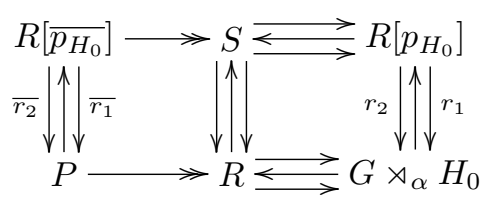

that is, all the commutative squares in the diagram are pullbacks. In particular $R$ and $R\left[p_{H_{0}}\right]$ are connected, $S$ is their centralizing double relation and the right hand side is the core of a profunctor (see [5]). Indeed, as the category is 
Barr-exact, $R$ is an effective equivalence relations on $G \rtimes_{\alpha} H_{0}$ and $S$ is effective on both $R\left[p_{H_{0}}\right]$ and $R$, so we can take their coequalizers and get an internal profunctor:

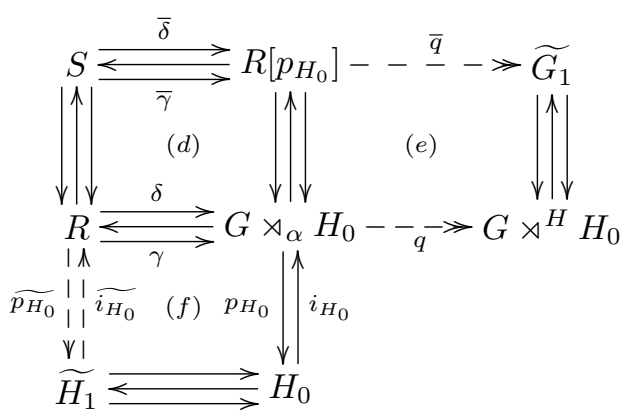

Since the leg $p_{H_{0}}$ is split epimorphic, this profunctor is representable and determines an internal functor of groupoids $\left(\widetilde{p_{1}}, \widetilde{p_{0}}\right)$, where $\widetilde{p_{0}}=q \cdot i_{H_{0}}$ and $\widetilde{p_{1}}=\bar{q} \cdot\left\langle 1, i_{H_{0}} p_{H_{0}}\right\rangle \cdot \gamma \cdot \widetilde{i_{H_{0}}}$ (see [18], Proposition 5.7). Moreover, by the universal property of the coequalizer $\overline{p_{H_{0}}}$, there is a morphism $h_{1}: H \rtimes_{\xi} H_{0} \rightarrow \widetilde{H_{1}}$, and $\left(h_{1}, 1_{H_{0}}\right)$ is a morphism of reflexive graphs. By composing with the given functor of groupoids we get a morphism of reflexive graphs:

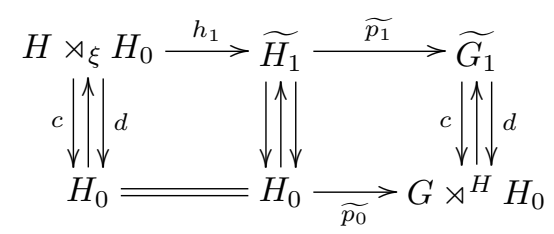

The following diagram shows that $p$ is the restriction of the arrow $\widetilde{p_{1}} \cdot h_{1}$ to the kernels of the codomain maps:

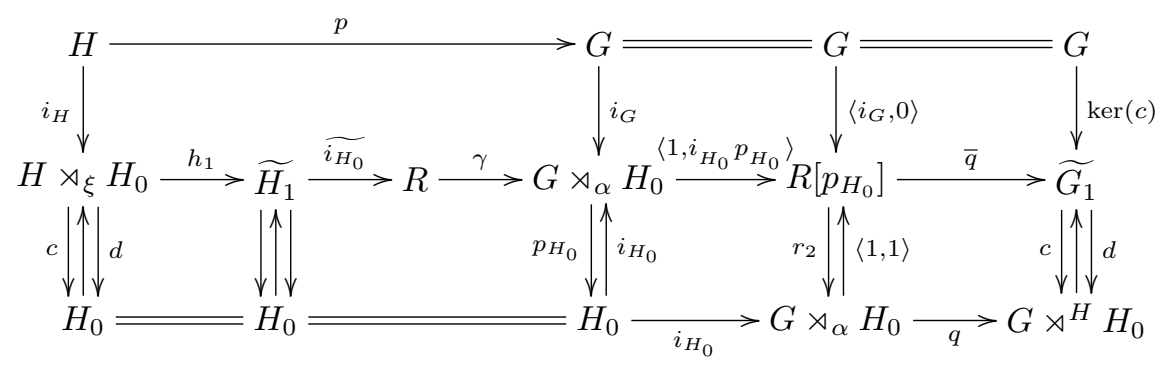

It is always possible to choose $\operatorname{ker}(c)$ in such a way that $\operatorname{ker}(c)=\bar{q} \cdot\left\langle 1, i_{H_{0}} p_{H_{0}}\right\rangle \cdot i_{G}$, so the only thing to prove is that $\gamma \cdot \widetilde{i_{H_{0}}} \cdot h_{1} \cdot i_{H}=i_{G} \cdot p$. But $\gamma \cdot \widetilde{i_{H_{0}}} \cdot h_{1}=\bar{c} \cdot \overline{i_{H_{0}}}=$ $r_{1} \cdot\langle 1,1\rangle \cdot(p \rtimes 1)=p \rtimes 1$ (see diagram (4)), hence $\gamma \cdot \widetilde{i_{H_{0}}} \cdot h_{1} \cdot i_{H}=(p \rtimes 1) \cdot i_{H}=i_{G} \cdot p$. As a consequence, the normalization of $\left(\widetilde{p_{1}}, \widetilde{p_{0}}\right)$ yields a morphism of pre-crossed 
modules which is the desired push forward:

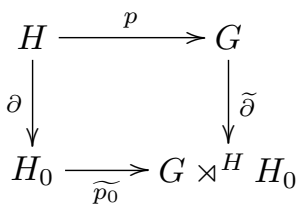

Now, combining the following pullbacks (the one on the right is part of $(e)$, while the left one is a commutative square where the two parallel split epimorphisms have isomorphic kernels):

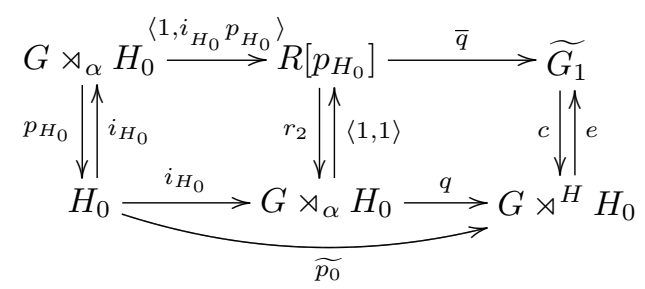

one gets that ${\widetilde{p_{0}}}^{*}(\widetilde{\xi})=\alpha$.

Finally, let us consider a morphism of pre-crossed modules

$$
\left(p, p_{0}\right):\left(\partial, \xi, H, H_{0}\right) \rightarrow\left(\partial^{\prime}, \xi^{\prime}, G, G_{0}\right),
$$

where $\left(\partial^{\prime}, \xi^{\prime}\right)$ is a crossed module, with $p_{0}^{*}\left(\xi^{\prime}\right)=\alpha$. This determines a representable profunctor $\left(p_{H_{0}}, q_{0}\right): \underline{\widetilde{H}} \rightarrow \underline{G}$ :

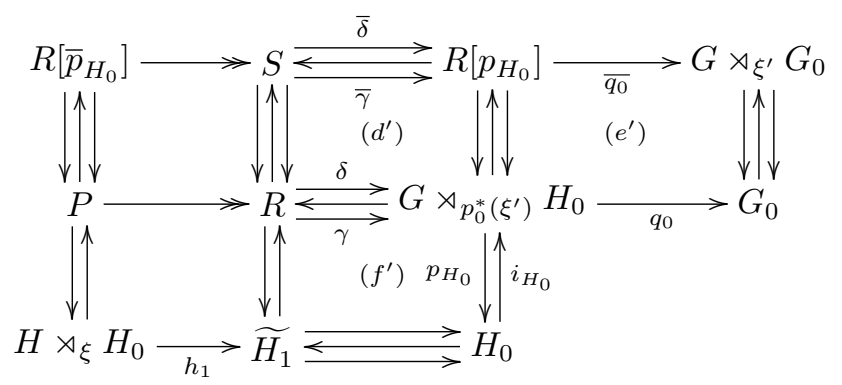

Since $p_{0}^{*}\left(\xi^{\prime}\right)=\alpha$, the square $\left(f^{\prime}\right)$ coincides with the square $(f)$, hence the square $\left(d^{\prime}\right)$ coincides with the square $(d)$. Moreover $q_{0}$ coequalizes $\delta$ and $\gamma$, similarly $\overline{q_{0}}$ coequalizes $\bar{\delta}$ and $\bar{\gamma}$. The property (PF) of the push forward easily follows from the universal property of the coequalizers $q$ and $\bar{q}$.

As a particular case, we recover the result of Proposition 3.4. 
Corollary 3.7. A pre-crossed module $(\partial, \xi)$ is a crossed module if and only if the following diagram commutes:

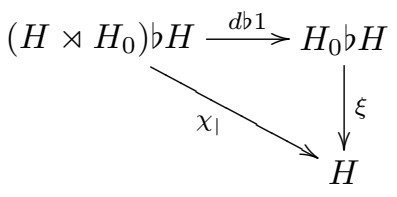

Proof. Apply Theorem 3.6 with $p=1_{H}$ and $\alpha=\xi$.

Remark 3.8. 1. If $H=0$, the conditions in Theorem 3.6 reduce to the request of existence of the action $\alpha$, and the above construction is nothing but a semi-direct product with its characterizing property (see Theorem 1.3 in $[12])$ :

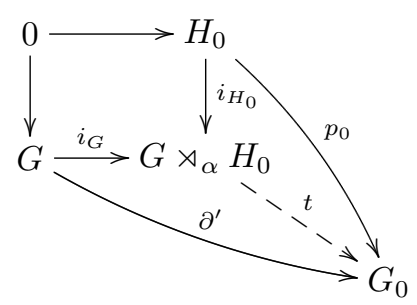

whence the use of the notation $G \rtimes^{H} H_{0}$.

2. In the case where $(\partial, \xi)$ is a crossed module, the push forward is a morphism of crossed modules. We call it push forward of crossed modules and we write $(\widetilde{\partial}, \widetilde{\xi})=p_{*}(\partial, \xi)$.

3. Whenever there exists a morphism of pre-crossed modules

$$
\left(p, p_{0}\right):\left(\partial, \xi, H, H_{0}\right) \rightarrow\left(\partial^{\prime}, \xi^{\prime}, G, G_{0}\right),
$$

with $\left(\partial^{\prime}, \xi^{\prime}\right)$ is a crossed module, it is always possible to compute the push forward of $\partial$ along $p$, with respect to the action $p_{0}^{*}\left(\xi^{\prime}\right): H_{0} b G \rightarrow G$. Indeed, the first condition for the construction of the push forward follows from the fact that $\left(p, p_{0}\right)$ is a pre-crossed module morphism, as the commutative diagram below shows:

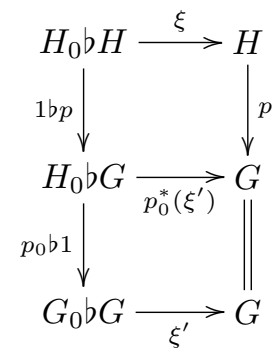


On the other hand, since $\left(\partial^{\prime}, \xi^{\prime}\right)$ is a crossed module, by Proposition 3.4, the following diagram is commutative:

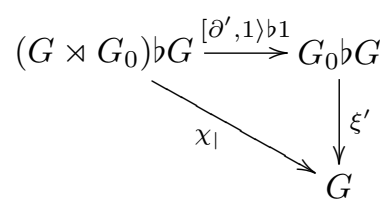

and by composition, we get the second condition of Theorem 3.6:

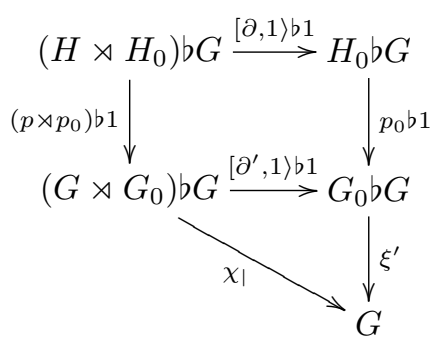

This also shows that the hypothesis of Theorem 3.6 are necessary.

\subsection{The case of action accessible categories}

In many algebraic contexts, the construction of the push forward can be performed under milder hypothesis on the arrows and the actions involved. In particular, we restrict our attention to semi-abelian action accessible categories (see [8]). Here the following result holds:

Lemma 3.9. In an action accessible category, let be given two subobjects of the same object:

$$
X \triangleright^{x}>Z<^{y}<Y,
$$

with $x$ a normal monomorphism. If $\bar{y}$ is the normal closure of $y$, then $x$ and $y$ cooperate if and only if $x$ and $\bar{y}$, in other words:

$$
[X, Y]=0 \Rightarrow[X, \bar{Y}]=0
$$

where $\bar{Y}$ is the normal closure of $Y$ in $Z$.

For details on cooperating morphisms and commutators see, for example, [2].

Proof. This property follows from the fact that, in action accessible categories, normal subobjects admit normal centralizers (see [8]).

In this case, we can state the same result of Theorem 3.6 with the weaker condition expressed by diagram (5) below. This is done by pre-composing diagram (3) with $i_{H} b 1: H b G \rightarrow\left(H \rtimes_{\xi} H_{0}\right) b G$. 
Theorem 3.10 (Push forward, action accessible case). In a semi-abelian action accessible category, we consider a pre-crossed module $\left(\partial: H \rightarrow H_{0}, \xi\right)$, a morphism $p: H \rightarrow G$, and an internal action $\alpha: H_{0} b G \rightarrow G$ such that the following diagrams commute:
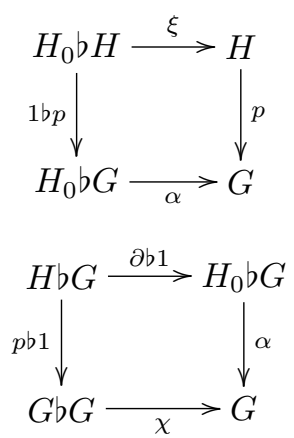

Then there exists a push forward $\left(\widetilde{\partial}: G \rightarrow G \rtimes^{H} H_{0}, \widetilde{\xi}\right)$, with the properties listed in Theorem 3.6.

Proof. First of all recall that, by definition of the conjugation action $\chi_{G}$, there is a well known isomorphism between $G \rtimes_{\chi} G$ and the product $G \times G$. Simply by composition, we get a similar situation for $G \rtimes_{p^{*}(\chi)} H$. More precisely, there is a unique arrow $[1, p\rangle$ making the following diagram commute:

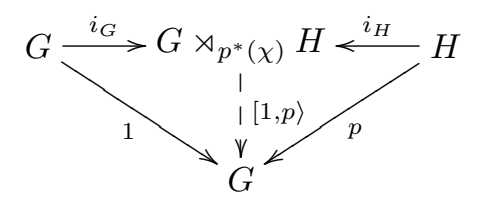

and we have an isomorphism $\tau=\left\langle[1, p\rangle, p_{H}\right\rangle$ of punctual spans:

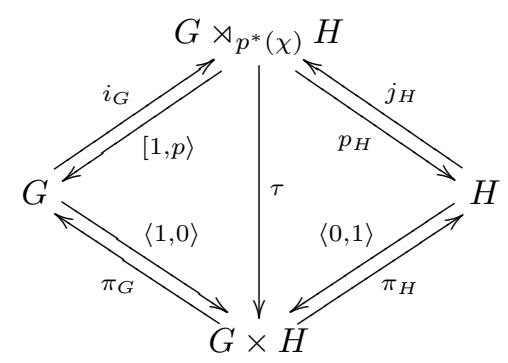

where $j_{H}=\operatorname{ker}[1, p\rangle$.

Since by hypothesis diagram (5) commutes, it follows that $p^{*}(\chi)=\partial^{*}(\alpha)$ and $G \rtimes_{p^{*}(\chi)} H=G \rtimes_{\partial^{*}(\alpha)} H$. 
Now, from the following commutative diagram

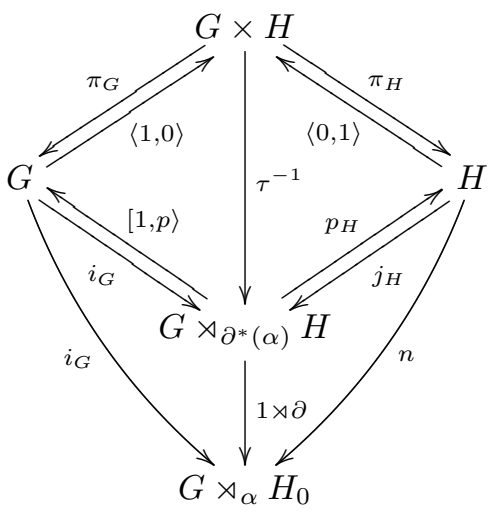

we deduce that the morphisms $n=(1 \rtimes \partial) \cdot j_{H}$ and $i_{G}$ cooperate in $G \rtimes_{\alpha} H_{0}$, so that $[n(H), G]=0$. Moreover, by Lemma 3.9:

$$
[n(H), G]=0 \quad \Rightarrow \quad[\overline{n(H)}, G]=0
$$

where $m: \overline{n(H)} \rightarrow G \rtimes_{\alpha} H_{0}$ is the normal closure of $n(H)$ in $G \rtimes_{\alpha} H_{0}$, and $h: H \rightarrow \overline{n(H)}$ is such that $m \cdot h=n$.

But, in the action accessible context, the so called "Smith is Huq" property holds, i.e. two normal subobjects cooperate if and only if the corresponding equivalence relations are connected. As a consequence, if $R$ is the equivalence relation corresponding to $\overline{n(H)}$, we have that $R$ and $R\left[p_{H_{0}}\right]$ are connected. Hence, by means of their centralizing double relation $S$, we get the core of a profunctor. Thus, we can apply the same technique as in the proof of Theorem 3.6 , obtaining a profunctor

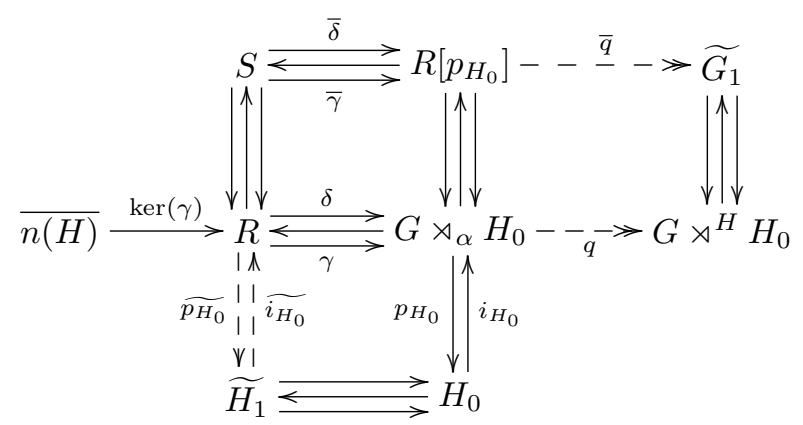

which represents the crossed module morphism on the right hand side of the 
following commutative diagram:

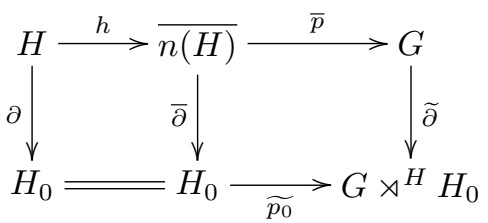

With an argument similar to the one used in the proof of Theorem 3.6, one can show that $\bar{p} \cdot h=p$. It remains to prove that the whole rectangle above is a morphism of pre-crossed modules, as the following commutative diagram shows (the arrow $p \rtimes 1$ exists by equivariance of $p$ ):

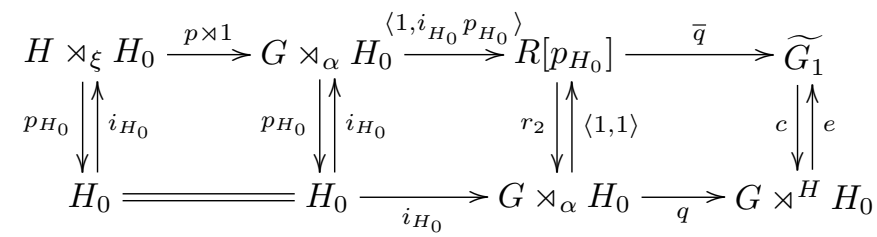

\subsection{A useful construction for the push forward of pre- crossed modules}

The proof of Theorem 3.6 takes place in the denormalized context of reflexive graphs, while the theorem itself is stated mainly in terms of pre-crossed modules (only diagram (3) uses the reflexive graph structure determined by the precrossed module $\partial$ ).

For this reason, it is useful to show a construction a posteriori, that takes place in the normalized context and may be used in applications. In fact, we will see how the object $G \rtimes^{H} H_{0}$ can be computed as a cokernel.

As in Section 3.2, we can consider the condition given by the commutativity of diagram (5) and the consequent isomorphism $G \rtimes_{\partial^{*}(\alpha)} H \cong G \times H$. In particular, we are interested in the arrow $(1 \rtimes \partial) \cdot j_{H}$.

Lemma 3.11. Under the hypothesis of Theorem 3.6, the composite

$$
H \stackrel{j_{H}}{\longrightarrow} G \rtimes_{\partial^{*}(\alpha)} H \stackrel{1 \rtimes \partial}{\longrightarrow} G \rtimes_{\alpha} H_{0}
$$

is a pre-crossed module. More precisely, it is the normalization $\bar{d} \cdot \operatorname{ker}(\bar{c})$ of the reflexive graph $\left(P, G \rtimes_{\alpha} H_{0}\right)$ introduced in the proof of the theorem.

Proof. Throughout this proof, we will refer to the notation and the diagrams used in the proof of Theorem 3.6 and $\operatorname{ker}(\bar{c})$ will be the unique arrow such that $\overline{p_{H_{0}}} \cdot \operatorname{ker}(\bar{c})=i_{H}: H \rightarrow H \rtimes H_{0}$. 
Consider the arrow $\psi$, defined by the universal property of the pullback $r_{2}$. $R(\bar{c})=\bar{c} \cdot \overline{r_{2}}$ :

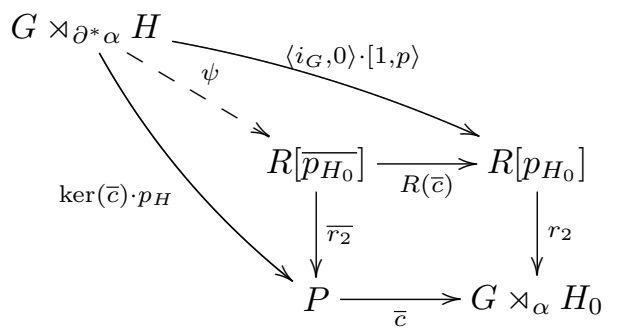

that is, $\psi$ is the unique arrow such that $\overline{r_{2}} \cdot \psi=\operatorname{ker}(\bar{c}) \cdot p_{H}$ and $R(\bar{c}) \cdot \psi=$ $\left\langle i_{G}, 0\right\rangle \cdot[1, p\rangle$.

First of all, we can prove that:

$$
\Delta_{P} \cdot \operatorname{ker}(\bar{c})=\psi \cdot j_{H}, \quad R(\bar{e}) \cdot\left\langle i_{G}, 0\right\rangle=\psi \cdot i_{G},
$$

where $R(\bar{e})$ is the common section of $R(\bar{d})$ and $R(\bar{c})$. To this end, it suffices to compose on the left with the jointly monic pair $\left(\overline{r_{2}}, R(\bar{c})\right)$.

The second step is to prove the equality:

$$
\overline{i_{H_{0}}} \cdot i_{H}=\overline{r_{1}} \cdot \psi \cdot i_{H},
$$

by composition on the left with the arrows $\overline{p_{H_{0}}}$ and $\bar{c}$, which are jointly monic as projections of the pullback $(b)+(c)$ in diagram (4).

Now, by composing on the right with the jointly epic pair $\left(i_{G}, i_{H}\right)$, we can prove that:

$$
1 \rtimes \partial=\bar{d} \cdot \overline{r_{1}} \cdot \psi .
$$

Finally we have:

$$
(1 \rtimes \partial) \cdot j_{H}=\bar{d} \cdot \overline{r_{1}} \cdot \psi \cdot j_{H}=\bar{d} \cdot \overline{r_{1}} \cdot \Delta_{P} \cdot \operatorname{ker}(\bar{c})=\bar{d} \cdot \operatorname{ker}(\bar{c})
$$

and the proof is completed.

As a consequence of Lemma 3.11, we have that $q=\operatorname{coker}\left((1 \rtimes \partial) \cdot j_{H}\right)$, since it is the coequalizer of $\bar{d}$ and $\bar{c}$, and this gives an alternative way to compute $G \rtimes^{H} H_{0}$.

Now, consider the following commutative diagram:

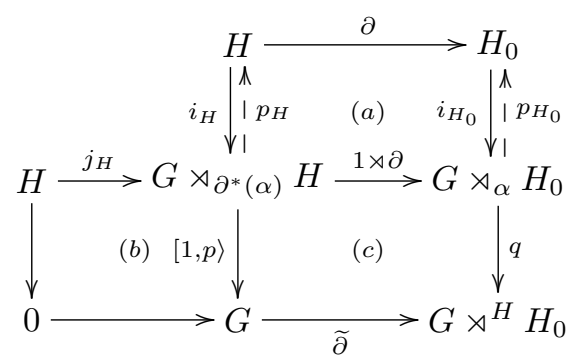


Since the sequence $\left(j_{H},[1, p\rangle\right)$ is short exact, the square $(b)$ is a pullback and a pushout. The rectangle $(b)+(c)$ is also a pushout, then also $(c)$ is a pushout. On the other hand, the pasting of $(a)$ and $(c)$ is the push forward of $\partial$ along $p=[1, p\rangle \cdot i_{H_{0}}$. Consequently, the push forward $\widetilde{\partial}$ can be alternatively obtained as the pushout of $1 \rtimes \partial$ along $p$.

It is also possible to describe the crossed module structure assigned to the map $\widetilde{\partial}$. This is done by observing that the action $\left(G \rtimes^{H} H_{0}\right) b G \rightarrow G$ is the only one making the following diagram commute:

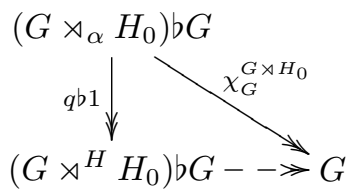

where the map $q b 1_{G}$ is a regular epimorphism since $q$ is and $-b 1_{G}$ preserves such maps (see [12]).

\subsection{A characterization}

Proposition 3.12. Let $\mathcal{C}$ be a semi-abelian category, and let

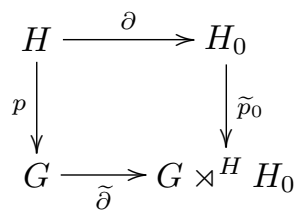

be a push forward of $\partial$ along $p$. Then the restriction $p_{\mid}$of $p$ to kernels is a regular epimorphism and

$$
\operatorname{Ker}\left(p_{\mid}\right)=\operatorname{Ker}(\partial) \cap \operatorname{Ker}(p) .
$$

Proof. We first decompose the push forward square into the rectangle $(a)$ and the rectangle $(c)$ of diagram (6). Since $(a)$ is a pullback, we can just consider the restriction of $[1, p\rangle$ to the kernels of the horizontal arrows in $(c)$.

We factorize the maps $1 \rtimes \partial$ and $\widetilde{\partial}$ into regular epimorphisms followed by monomorphisms. We get the commutative diagram:

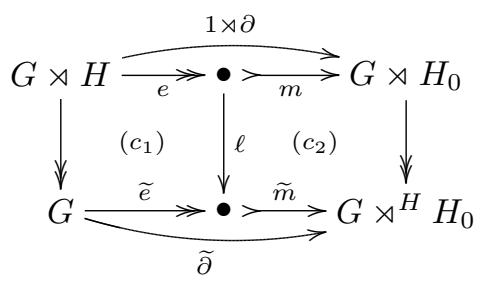

where the comparison map $\ell$ is a regular epimorphism. 
Let us consider the diagram below:

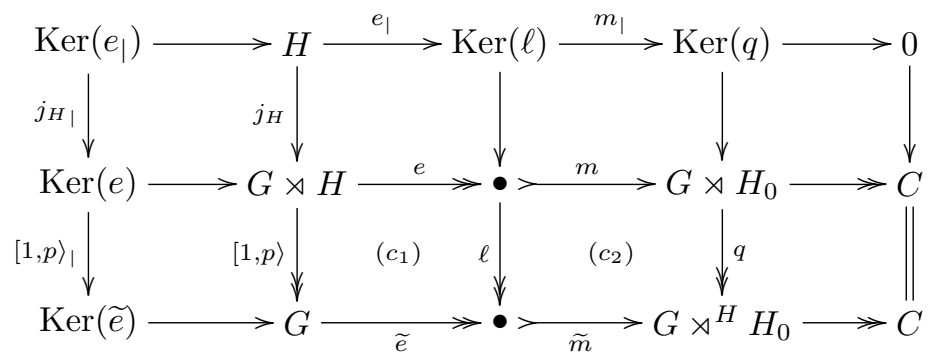

which extends $\left(c_{1}\right)+\left(c_{2}\right)$ in the following way. First we take the kernels of the (vertical) arrows $[1, p\rangle, \ell$ and $q$, and consider the restrictions $e_{\mid}$and $m_{\mid}$of $e$ and $m$ respectively. Since $m \cdot e \cdot j_{H}=(1 \rtimes \partial) \cdot j_{H}$ is a pre-crossed module (see Lemma 3.11), it factorizes into a regular epimorphism followed by a normal monomorphism, the last being the kernel of $\operatorname{coker}\left(m \cdot e \cdot j_{H}\right)=q$. So the composition $m_{\mid} \cdot e_{\mid}$is a regular epimorphism.

Then we observe that the monomorphisms $m$ and $\widetilde{m}$ are normal, since they come from a factorization of two pre-crossed modules, and they have isomorphic cokernels just because $\left(c_{1}\right)+\left(c_{2}\right)$ is a pushout. This allows us to complete the diagram with the last column on the right, in such a way that the four squares on the right commute. In fact, in the four squares on the right, the three vertical sequences and the bottom and middle horizontal sequences are short exact. The $3 \times 3$ lemma (see e.g. [2]) ensures that also the upper horizontal sequence is short exact, so that $m_{\mid}$is an isomorphism.

On the other hand, we already observed that the composition $m_{\mid} \cdot e_{\mid}$is a regular epimorphism, and then $e_{\mid}$itself is a regular epimorphism. This implies immediately that the square $\left(c_{1}\right)$ is a pushout.

Now we can take the kernels of $e, \widetilde{e}$ and $e_{\mid}$. Call $j_{H \mid}$ and $[1, p\rangle_{\mid}$the restrictions of $j_{H}$ and $[1, p\rangle$ respectively. By applying $3 \times 3$ lemma again, since the horizontal sequences are short exact likewise the middle and the rightmost vertical ones, we can conclude that also the leftmost vertical sequence is short exact.

This fact concludes the proof. Indeed $[1, p\rangle_{\mid}$, and then $p_{\mid}$, are regular epimorphisms, and $\operatorname{Ker}\left(p_{\mid}\right)=\operatorname{Ker}\left(e_{\mid}\right)=\operatorname{Ker}(e) \cap H=\operatorname{Ker}(1 \rtimes \partial) \cap \operatorname{Ker}([1, p\rangle)=$ $\operatorname{Ker}(\partial) \cap \operatorname{Ker}(p)$.

Theorem 3.13. Let $\mathcal{C}$ be a semi-abelian category, and let

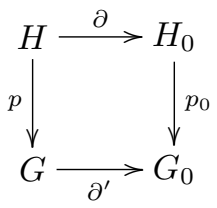

be a pre-crossed module morphism, with $\left(\partial^{\prime}, \xi^{\prime}\right)$ a crossed module.

Then $\left(p, p_{0}\right)$ makes $\partial^{\prime}$ the push forward of $\partial$ along $p$ with respect to the action $p_{0}^{*}\left(\xi^{\prime}\right)$ if and only if it induces an isomorphism between the cokernels and a regular epimorphism between the kernels of $\partial$ and $\partial^{\prime}$. 
Proof. The only if part is a consequence of Theorem 3.6 (2) and Proposition 3.12 above.

For what concerns the other implication, thanks to Remark 3.8 (3), we can compute the push forward $\widetilde{\partial}$ of $\partial$ along $p$ with respect to the action $p_{0}^{*}\left(\xi^{\prime}\right)$. By comparison, we obtain a factorization of $\left(p, p_{0}\right)$ which is represented by the two squares on the right in the diagram below. Then we take the kernels of $\partial, \widetilde{\partial}$ and $\partial^{\prime}$, and we complete the diagram with the restrictions $p_{\mid}$and $k^{\prime}$ :

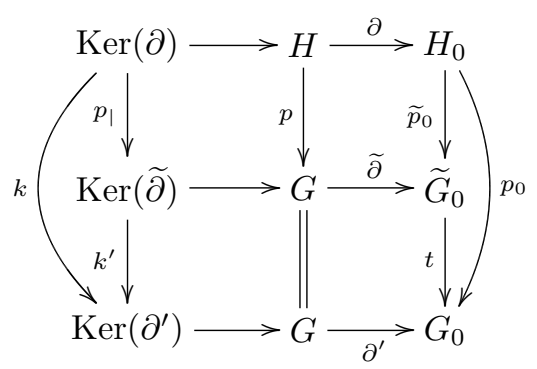

Since $k=k^{\prime} \cdot p$ is a regular epimorphism by hypothesis, then also $k^{\prime}$ is. But $k^{\prime}$ is also a monomorphism, hence it is an isomorphism.

Now, we have proved that $\widetilde{\partial}$ and $\partial^{\prime}$ have isomorphic kernels, so that they factor through the same (isomorphic) cokernel(s), i.e. the regular epimorphism $c$ in the commutative diagram below:

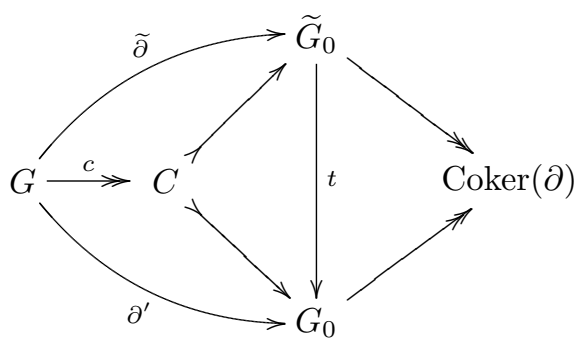

Since $\widetilde{\partial}$ and $\partial^{\prime}$ are crossed modules, the two images through $c$ are normal, so that the two triangles on the right form a morphism of short exact sequences. By the short five lemma, $t$ is an isomorphism.

Corollary 3.14. Push forwards are closed under composition.

Proof. Trivial by Theorem 3.13.

\section{Examples}

In this section we present some examples of explicit computation of the push forward in algebraic varieties (see [20] for the case of groups). The examples below take place in action accessible categories, so we will refer to the conditions given in Theorem 3.10. Moreover, following [13], internal actions can be described by appropriate set-theoretical functions satisfying suitable conditions. 


\section{$4.1 \quad$ Rings}

Following the notation of Theorem 3.10, let $\left(\partial: H \rightarrow H_{0}, \xi\right)$ be a pre-crossed module in the category of rings. The action $\xi$ can be given by the assignment of two bilinear maps:

$$
\begin{aligned}
H_{0} \times H & \rightarrow H, & \left(h_{0}, h\right) & \mapsto h_{0} \cdot h, \\
H \times H_{0} & \rightarrow H, & \left(h, h_{0}\right) & \mapsto h \cdot h_{0},
\end{aligned}
$$

satisfying the following identities (for all $h, h^{\prime} \in H$ and $h_{0}, h_{0}^{\prime} \in H_{0}$ ):

$$
\begin{array}{ll}
\left(h_{0} h_{0}^{\prime}\right) \cdot h=h_{0} \cdot\left(h_{0}^{\prime} \cdot h\right), & \left(h_{0} \cdot h\right) h^{\prime}=h_{0} \cdot\left(h h^{\prime}\right), \\
\left(h_{0} \cdot h\right) \cdot h_{0}^{\prime}=h_{0} \cdot\left(h \cdot h_{0}^{\prime}\right), & \left(h \cdot h_{0}\right) h^{\prime}=h\left(h_{0} \cdot h^{\prime}\right), \\
\left(h \cdot h_{0}\right) \cdot h_{0}^{\prime}=h \cdot\left(h_{0} h_{0}^{\prime}\right), & \left(h h^{\prime}\right) \cdot h_{0}=h\left(h^{\prime} \cdot h_{0}\right) .
\end{array}
$$

The pre-crossed module condition says that:

$$
\partial\left(h_{0} \cdot h\right)=h_{0} \cdot \partial(h) \quad \text { and } \quad \partial\left(h \cdot h_{0}\right)=\partial(h) \cdot h_{0} .
$$

In the same way, let an action $\alpha$ of $H_{0}$ on $G$ be given, together with a ring homomorphism $p: H \rightarrow G$, equivariant with respect to the actions $\xi$ and $\alpha$, i.e. such that for all $h \in H$ and $h_{0} \in H_{0}$ :

$$
p\left(h_{0} \cdot h\right)=h_{0} \cdot p(h) \quad \text { and } \quad p\left(h \cdot h_{0}\right)=p(h) \cdot h_{0} .
$$

Moreover, the condition given by diagram (5) requires that $p^{*}\left(\chi_{G}\right)=\partial^{*}(\alpha)$, i.e. for all $h \in H$ and $g \in G$ :

$$
\partial(h) \cdot g=p(h) g \quad \text { and } \quad g \cdot \partial(h)=g p(h) .
$$

Under these hypothesis, we can compute the push forward of $\partial$ along $p$ in the following way.

Consider the semi-direct product $G \rtimes_{\alpha} H_{0}$, which is given by the set $G \times H_{0}$ endowed with the operations:

$$
\begin{aligned}
& \left(g, h_{0}\right)+\left(g^{\prime}, h_{0}^{\prime}\right)=\left(g+g^{\prime}, h_{0}+h_{0}^{\prime}\right) \\
& \left(g, h_{0}\right) \cdot\left(g^{\prime}, h_{0}^{\prime}\right)=\left(g g^{\prime}+h_{0} \cdot g^{\prime}+g \cdot h_{0}^{\prime}, h_{0} h_{0}^{\prime}\right)
\end{aligned}
$$

and the ring homomorphism:

$$
n=(1 \rtimes \partial) \cdot j_{H}: H \rightarrow G \rtimes H_{0}, \quad h \mapsto(-p(h), \partial(h)) .
$$

The image $n(H)$ is an ideal of $G \rtimes H_{0}$ since, for all $h \in H, g \in G$ and $h_{0} \in H_{0}$ :

$$
\begin{aligned}
& \left(g, h_{0}\right) \cdot(-p(h), \partial(h))=\left(-g p(h)-h_{0} \cdot p(h)+g \cdot \partial(h), h_{0} \partial(h)\right)=\left(-p\left(h_{0} \cdot h\right), \partial\left(h_{0} \cdot h\right)\right), \\
& (-p(h), \partial(h)) \cdot\left(g, h_{0}\right)=\left(-p(h) g+\partial(h) \cdot g-p(h) \cdot h_{0}, \partial(h) h_{0}\right)=\left(-p\left(h \cdot h_{0}\right), \partial\left(h \cdot h_{0}\right)\right) .
\end{aligned}
$$

So we can take the quotient homomorphism:

$$
q: G \rtimes H_{0} \rightarrow G \rtimes^{H} H_{0}=\left(G \rtimes H_{0}\right) / n(H) .
$$


Since the conjugation action of $n(H)$ on $G$ is trivial:

$$
\begin{aligned}
& (g, 0) \cdot(-p(h), \partial(h))=(-g p(h)+g \cdot \partial(h), 0)=(0,0), \\
& (-p(h), \partial(h)) \cdot(g, 0)=(-p(h) g+\partial(h) \cdot g, 0)=(0,0),
\end{aligned}
$$

the conjugation action of $G \rtimes H_{0}$ on $G$ is well defined on cosets, thus giving an action of $G \rtimes^{H} H_{0}$ on $G$. This is the action $\widetilde{\xi}$ making the morphism $q \cdot i_{G}=\widetilde{\partial}$ a crossed module.

\subsection{Lie and Leibniz algebras}

Consider now the category of Lie algebras over a fixed field. As above, $\partial$ is a pre-crossed module. Here, the action $\xi$ is a bilinear map:

$$
H_{0} \times H \rightarrow H, \quad\left(h_{0}, h\right) \mapsto\left[h_{0}, h\right]
$$

satisfying the following identities (for all $h, h^{\prime} \in H$ and $h_{0}, h_{0}^{\prime} \in H_{0}$ ):

$$
\begin{aligned}
& {\left[\left[h_{0}, h_{0}^{\prime}\right], h\right]=\left[h_{0},\left[h_{0}^{\prime}, h\right]\right]-\left[h_{0}^{\prime},\left[h_{0}, h\right]\right]} \\
& {\left[h_{0},\left[h, h^{\prime}\right]\right]=\left[\left[h_{0}, h\right], h^{\prime}\right]-\left[\left[h_{0}^{\prime}, h^{\prime}\right], h\right]}
\end{aligned}
$$

and the pre-crossed module condition says that:

$$
\partial\left[h_{0}, h\right]=\left[h_{0}, \partial(h)\right] .
$$

An action $\alpha$ of $H_{0}$ on $G$ and a Lie algebra homomorphism $p: H \rightarrow G$ are given, and the equivariance of $p$ says that for all $h \in H$ and $h_{0} \in H_{0}$ :

$$
p\left[h_{0}, h\right]=\left[h_{0}, p(h)\right] .
$$

Finally, the request $p^{*}\left(\chi_{G}\right)=\partial^{*}(\alpha)$ means that for all $h \in H$ and $g \in G$ :

$$
[\partial(h), g]=[p(h), g] .
$$

Under these hypothesis, we can compute the push forward of $\partial$ along $p$ as above. The semi-direct product $G \rtimes_{\alpha} H_{0}$ is given by the set $G \times H_{0}$ endowed with the operations:

$$
\begin{aligned}
& \left(g, h_{0}\right)+\left(g^{\prime}, h_{0}^{\prime}\right)=\left(g+g^{\prime}, h_{0}+h_{0}^{\prime}\right) \\
& {\left[\left(g, h_{0}\right),\left(g^{\prime}, h_{0}^{\prime}\right)\right]=\left(\left[g, g^{\prime}\right]+\left[h_{0}, g^{\prime}\right]-\left[h_{0}^{\prime}, g\right],\left[h_{0}, h_{0}^{\prime}\right]\right)}
\end{aligned}
$$

and we have the homomorphism:

$$
n=(1 \rtimes \partial) \cdot j_{H}: H \rightarrow G \rtimes H_{0}, \quad h \mapsto(-p(h), \partial(h)) .
$$

The image $n(H)$ is an ideal of $G \rtimes H_{0}$, indeed, for all $h \in H, g \in G$ and $h_{0} \in H_{0}$ :

$\left[\left(g, h_{0}\right),(-p(h), \partial(h))\right]=\left(-[g, p(h)]-\left[h_{0}, p(h)\right]-[\partial(h), g],\left[h_{0}, \partial(h)\right]\right)=\left(-p\left[h_{0}, h\right], \partial\left[h_{0}, h\right]\right)$

and we can take the quotient homomorphism:

$$
q: G \rtimes H_{0} \rightarrow G \rtimes^{H} H_{0}=\left(G \rtimes H_{0}\right) / n(H) .
$$


Since the conjugation action of $n(H)$ on $G$ is trivial:

$$
[(-p(h), \partial(h)),(g, 0)]=(-[p(h), g]+[\partial(h), g], 0)=(0,0)
$$

again, there is a well defined action of $G \rtimes^{H} H_{0}$ on $G$ making the morphism $q \cdot i_{G}$ a crossed module.

In the case of Leibniz algebras (see [14] for definitions), we still have a bracket operation, satisfying a weaker version of the Jacobi identity, and which is not antisymmetric in general. As a consequence, actions are given by pairs of bilinear maps, likewise in the case of rings, and the conditions involving actions are a bit more complicated. However the homomorphism $n$ is defined exactly as above and the push forward construction is the same.

\section{Push forward of extensions}

\subsection{Abelian extensions}

Let $\mathcal{C}$ be a semi-abelian category. Given an object $Y$ in $\mathcal{C}$, following Beck's terminology (see [1]), we call $Y$-module an abelian group object in the slice category $\mathcal{C} \downarrow Y$, i.e. a totally disconnected groupoid in $\mathcal{C}$ with $Y$ as object of objects:

$$
A \rtimes_{\xi} Y \underset{p_{Y}}{\stackrel{p_{Y}}{\gtrless-i_{Y} \longrightarrow}} Y
$$

A $Y$-module morphism is then an internal functor of the form:

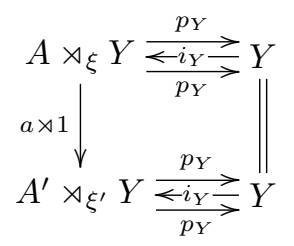

which is uniquely determined by a morphism $a: A \rightarrow A^{\prime}$ in $\mathcal{C}$ such that:

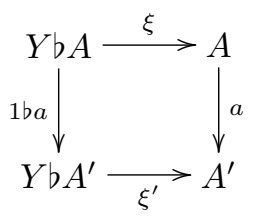

Classically, a $Y$-module is simply an abelian group object in $\mathcal{C}$, together with an action $\xi$ of $Y$ on $A$. In general, this notion is weaker than the one given above; however, if the "Smith is Huq" property holds (as, for example, in action accessible categories), the two definitions are equivalent. On the other hand, the notion of $Y$-module morphism remains the same. 
Now, consider an abelian extension in $\mathcal{C}$, that is a short exact sequence:

$$
0 \longrightarrow A \stackrel{i}{\longrightarrow} X \stackrel{f}{\longrightarrow} Y \longrightarrow 0
$$

with $R[f]$ connected with itself. In other words, $f$ is a Mal'tsev object in $\mathcal{C} \downarrow Y$ (see e.g. [2]). Again, if the "Smith is Huq" property holds, this is the same as an extension with abelian kernel. Such an extension is associated with a $Y$-module structure on $A$, defined as the direction of the Mal'tsev object [4].

Proposition 5.1. Given an abelian extension $f$ as above, with direction $(A, \xi)$, a $Y$-module $\left(A^{\prime}, \xi^{\prime}\right)$ and a morphism $a:(A, \xi) \rightarrow\left(A^{\prime}, \xi^{\prime}\right)$ of $Y$-modules, then there exists an abelian extension:

$$
0 \longrightarrow A^{\prime} \stackrel{i^{\prime}}{\longrightarrow} X^{\prime} \stackrel{f^{\prime}}{\longrightarrow} Y \longrightarrow 0
$$

with direction $\left(A^{\prime}, \xi^{\prime}\right)$, and a morphism of extensions:

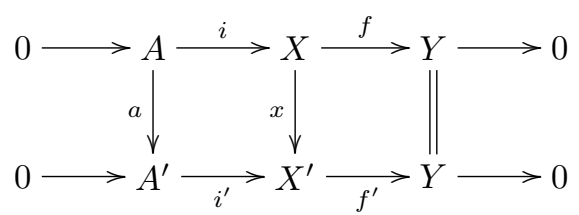

Proof. It suffices to show that the push forward of $i$ along $a$ exists (with respect to the action $f^{*}\left(\xi^{\prime}\right): X \triangleright A^{\prime} \rightarrow A^{\prime}$ ), and construct in such a way the square $x \cdot i=i^{\prime} \cdot a$. Indeed, if it is the case, Theorem 3.13 shows that the crossed module morphism $(a, x)$ induces an isomorphism between the cokernels and a regular epimorphism between the kernels. Hence, $\operatorname{Coker}\left(i^{\prime}\right)=Y$ and $\operatorname{Ker}\left(i^{\prime}\right)=0$, so that $i^{\prime}$ is a monomorphism (and it is normal, being a crossed module).

So, we only have to prove that the conditions for the construction of the push forward are fulfilled. The equivariance of $a$ comes from the fact that $a$ is a morphism of $Y$-modules:

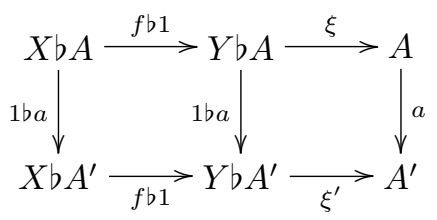

To check the second condition, we have to prove the commutativity of the 
following diagram:

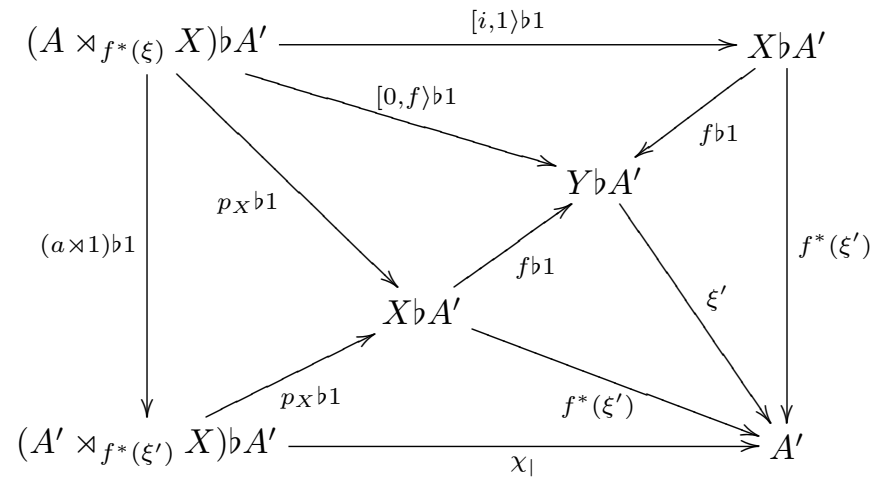

where the only commutativity which is not obvious is the one of the lower triangle $f^{*}\left(\xi^{\prime}\right) \cdot p_{X} b 1=\chi_{\mid}$. This equality depends on the fact that $A^{\prime}$ is a $Y$-module, so that the groupoid

$$
A^{\prime} \rtimes_{f^{*}\left(\xi^{\prime}\right)} X \underset{p_{X}}{\stackrel{p_{X}}{\underset{p_{X}}{\gtrless}}} X
$$

coincides with its direction. Hence, following the construction of [4], we have a dicrete fibration:

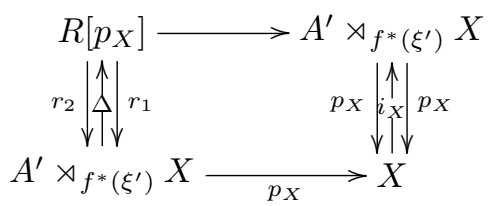

and this implies that $f^{*}\left(\xi^{\prime}\right) \cdot p_{X} b 1=\chi_{\mid}$.

Starting from a $Y$-module morphism $a$, the above proposition allows us to define a functor between groupoids:

$$
a_{*}: \operatorname{OPEXT}(Y, A, \xi) \rightarrow \operatorname{OPEXT}\left(Y, A^{\prime}, \xi^{\prime}\right)
$$

so that we can denote $f^{\prime}=a_{*}(f)$. It is also possible to prove that this construction respects Baer sums (since they are both push forwards), thus producing a group homomorphism:

$$
a_{*}: \operatorname{Opext}(Y, A, \xi) \rightarrow \operatorname{Opext}\left(Y, A^{\prime}, \xi^{\prime}\right),
$$

where Opext is the set of isomorphism classes of OPEXT, endowed with an abelian group structure given by Baer sums.

This way, we recover the fact, already proved in [7], that the assignment:

$$
\operatorname{Opext}_{\mathcal{C}}(Y,-):[\text { Abelian objects in } \mathcal{C} \downarrow Y] \rightarrow[\text { Abelian groups }]
$$


is functorial.

Moreover, since the extension $f^{\prime}$ is obtained by means of a push forward, it inherits the following property: for any other morphism of extensions of the form

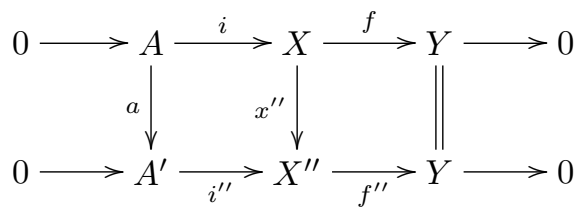

with $\left(A^{\prime}, \xi^{\prime}\right)$ direction of $f^{\prime \prime}$, there is a unique arrow $t$ such that $t \cdot x=x^{\prime \prime}$ and $t \cdot i^{\prime}=i^{\prime \prime}$ :

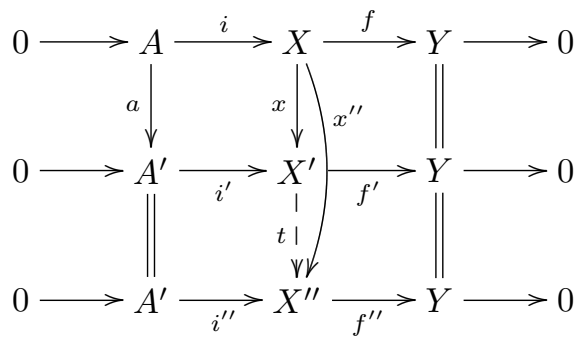

So we recover the fact that the direction functor is a pseudo cofibration, as already proved in [4] in a more general situation.

\subsection{General case}

In the previous section, we showed that a morphism $a$ of $Y$-modules is sufficient to produce the push foward of an abelian extension, providing a way to lift the morphism $a$ to a morphism of abelian extensions.

But the push forward construction holds, under suitable hypothesis, also for general (not necessarily abelian) extensions, and Theorem 3.13 ensures that the push forward of an extension is again an extension. So, the conditions (2) and (3) of Theorem 3.6, in the special case where $\partial$ is a normal monomorphism, can be regarded as conditions to generalize the above construction. More precisely, the following result holds.

Proposition 5.2. Given an extension:

$$
0 \longrightarrow K \stackrel{i}{\longrightarrow} X \stackrel{f}{\longrightarrow} Y \longrightarrow 0
$$

a morphism $k: K \rightarrow K^{\prime}$ and an action $\alpha$ of $X$ on $K^{\prime}$, such that the following diagrams commute:

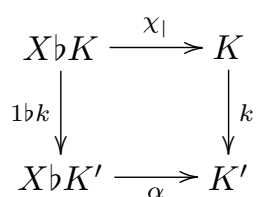




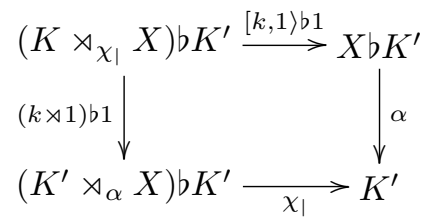

then there exists an extension:

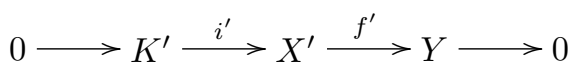

and a morphism of extensions:

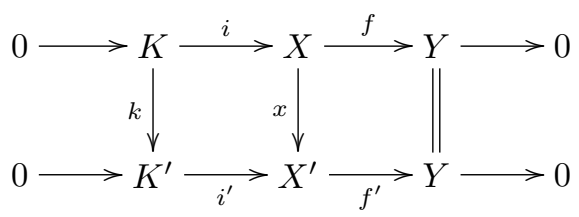

\section{Push forward and the comprehensive factor- ization for internal crossed modules}

In this section we will establish a link between the push forward construction developed in Section 3 and the comprehensive factorization system in $\operatorname{XMod}(\mathcal{C})$, for $\mathcal{C}$ a semi-abelian category.

\subsection{Factorization systems for internal crossed modules}

Factorization systems for internal groupoids have been studied in [3, 9], so that it is quite natural to express them in terms of crossed modules.

Let $\mathcal{C}$ be a Barr-exact category. In [3] the author studies two factorization systems for $\mathbf{G p d}(\mathcal{C})$, and he shows the way they are related to each other.

The first one consists of the classes of $\pi_{0}$-invertible and $\pi_{0}$-cartesian morphisms, where the reflection

$$
\pi_{0}: \operatorname{Gpd}(\mathcal{C}) \longrightarrow \mathcal{C}
$$

functorially extends the assignment $\pi_{0}(\underline{G})=\operatorname{Coeq}\left(d_{\underline{G}}, c_{\underline{G}}\right)$, for any groupoid $\underline{G}$. Indeed, $\pi_{0}$ is a fibration up to isomorphism (fibred reflection in [3]), and its definition, rather than being an ad hoc one, comes from a universal construction. Actually, the category $\mathbf{G p d}(\mathcal{C})$ is monadic over the category $\operatorname{Pt}(\mathcal{C})$ (of split epimorphisms with a chosen section) and $\pi_{0}$ is the extension to the algebras of the forgetful functor

$$
()_{0}: \operatorname{Pt}(\mathcal{C}) \longrightarrow \mathcal{C},
$$

that sends a split epimorphism $p$, with section $s$, to the codomain of $p$.

The second factorization system consists of the classes $D$ and $F$ of discrete fibrations and of final functors respectively, and it is the lifting to the algebras of the factorization system determined on $\mathbf{P t}(\mathcal{C})$ by the fibration ()$_{0}$. 
For what concerns the first factorization system, the constructions involved in the case of groupoids are fairly easy to translate. Actually, the main fact is that, for a groupoid $\underline{G}$, the coequalizer $\pi_{0}(\underline{G})$ amounts to the cokernel of the map underlying the corresponding crossed module $\left(\partial_{\underline{G}}, \xi_{\underline{G}}\right)$. Thanks to this fact, one simply translates the problem in terms of groupoids, then one normalizes the resulting construction. The outcome is a factorization of a given morphism of crossed modules

$$
\left(f, f_{0}\right): \underline{G} \rightarrow \underline{H}
$$

described in the diagram below:

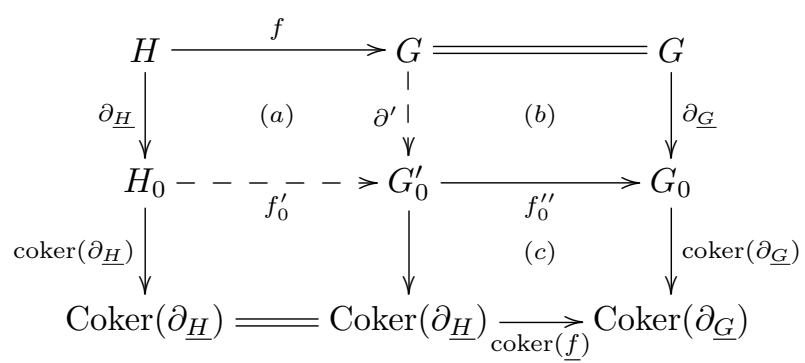

Here the bottom right square $(c)$ is a pullback by construction, so that the comparison map $f_{0}^{\prime}$ gives $f_{0}^{\prime \prime} f_{0}^{\prime}=f_{0}$. Moreover, it can be shown that the comparison map $\partial^{\prime}=\left\langle 0, \partial_{G}\right\rangle$ inherits a crossed module structure, such that $(a)$ and $(b)$ are morphisms, and the vertical unlabelled arrow is the cokernel of $\partial^{\prime}$.

Hence, we obtain the desired factorization of $\left(f, f_{0}\right)$ into the $\pi_{0}$-invertible morphism $\left(f, f_{0}^{\prime}\right)$ followed by the $\pi_{0}$-cartesian morphism $\left(1_{G}, f_{0}^{\prime \prime}\right)$.

\subsection{Final morphisms of internal crossed modules}

The comprehensive factorization of a functor has been introduced in the settheoretical context by Street and Walters in [23]. An internal version has been developed by Bourn in [3] for internal groupoids in a Barr-exact category, further extended in [9] to the efficiently regular setting.

The notion of final morphism of crossed modules we are going to introduce is a mere translation of the one defined by Bourn. Nevertheless, the technique adopted here is different: indeed, it arises from the property $(\mathrm{PF})$ of push forward.

Let us recall from [3] that, for a Barr-exact category $\mathcal{C}$, the class of final functors is the class $F$ of all internal functors $\underline{h}: \underline{H} \rightarrow \underline{G}$ satisfying the following property: for any commutative diagram (of solid arrows) in $\mathbf{G p d}(\mathcal{C})$

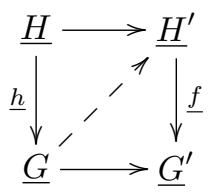


with $f$ a discrete fibration, there exists a (dashed) diagonal such that both triangles above commute. The pair $(F, D)$ constitutes a factorization system.

The notion of discrete fibration can be easily translated in terms of crossed module morphism: a morphism $f=\left(f, f_{0}\right)$ is (i.e. it corresponds to) a discrete fibration if and only if $f$ is an isomorphism.

For what concerns final morphisms, they are clearly defined by the diagonalization property as above, but in order to make this notion easier to handle, we are going to give a characterization.

Proposition 6.1. Let $\mathcal{C}$ be a semi-abelian category and let $\underline{G}=\left(\partial_{G}: G \rightarrow\right.$ $\left.G_{0}, \xi_{G}\right)$ and $\underline{H}=\left(\partial_{H}: H \rightarrow H_{0}, \xi_{H}\right)$ be crossed modules.

The following conditions are equivalent for a morphism $\underline{f}=\left(f, f_{0}\right): \underline{H} \rightarrow \underline{G}$ :

1. $f$ is final;

2. $f$ is obtained as a push forward of $\partial_{H}$ along $f$, w.r.t. the induced action

$$
H_{0} b G \stackrel{f_{0} b 1}{\longrightarrow} G_{0} b G \stackrel{\xi_{G}}{\longrightarrow} G .
$$

Proof. First we prove the implication $(2) \Rightarrow(1)$. Let $f$ be obtained as a push forward. We can factorize it as a final functor followed by a discrete fibration:

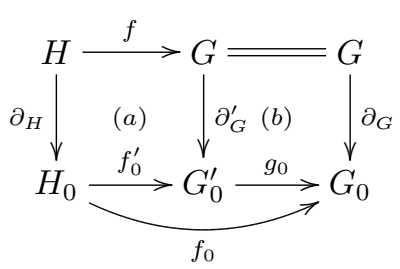

where the action of the crossed module $\partial_{G}^{\prime}$ is univocally determined by the (equivariance of the) discrete fibration $(b)$, namely:

$$
\xi_{G}^{\prime}: G_{0}^{\prime} b G \stackrel{g_{0} b 1}{\longrightarrow} G_{0} b \stackrel{\xi_{G}}{\longrightarrow} G .
$$

Actually, $\left(1_{G}, g_{0}\right)$ is an isomorphism of crossed modules.

In order to prove this statement, we can start by computing the push forward inside the square $(a)$ with respect to the induced action $\xi_{G}^{\prime} \cdot\left(f_{0}^{\prime} b 1\right)$ (see Remark 3.8). Indeed,

$$
\xi_{G}^{\prime} \cdot\left(f_{0}^{\prime} b 1\right)=\xi_{G} \cdot\left(g_{0} b 1\right) \cdot\left(f_{0}^{\prime} b 1\right)=\xi_{G} \cdot\left(f_{0} b 1\right),
$$

so that this push forward gives the same morphism $f$ we started with. This yields the following factorization:

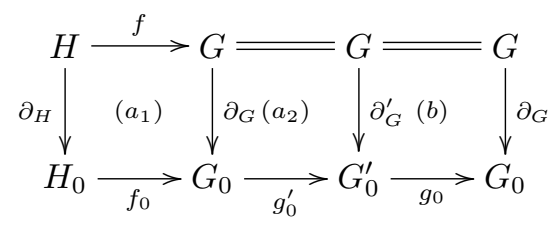


By the main property of the push forward $\left(a_{1}\right)$, then $\left(a_{2}\right)+(b)$ must give the identity of $\underline{G}$.

Again we can factorize $\left(a_{1}\right)=(a)+(b)$ and get

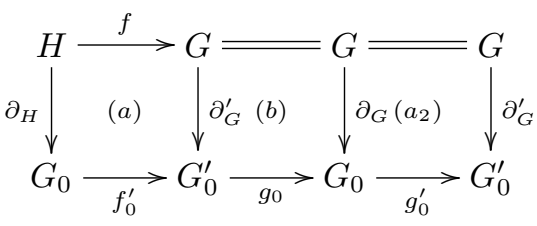

By the essential uniqueness of the factorization, the discrete fibration $(b)+\left(a_{2}\right)$ must be the identity of $\underline{G}^{\prime}$, showing that $g_{0}$ is an isomorphism.

Now, let $f$ be a final functor (notation as above). Indeed, it is also the push forward of $\partial_{H}$ along the map $f$, with respect to the induced action. This is easily proved by performing the push forward. Since this is also a final functor by the first implication and since the comparison is a discrete fibration, the result follows from the essential uniqueness of the factorization.

The following corollary is a straightforward application of Proposition 3.13.

Corollary 6.2. A morphism of crossed modules $f: \underline{H} \rightarrow \underline{G}$ is final if and only if it induces an isomorphism between the cokernels of $\partial_{\underline{H}}$ and $\partial_{\underline{G}}$ and a regular epimorphism between the kernels of $\partial_{\underline{H}}$ and $\partial_{\underline{G}}$.

As a consequence, if we define the kernel functor Ker: $\operatorname{XMod}(\mathcal{C}) \rightarrow \mathcal{C}$ as:

$$
\underline{G} \mapsto \operatorname{Ker}\left(\partial_{\underline{G}}\right),
$$

it is easy to see that Ker turns the (final functor, discrete fibration) factorization of a given morphism $g$ into the (regular epi, mono) factorization of $\operatorname{Ker}(\underline{g})$.

Remark 6.3. It is interesting to state the denormalized version of the last corollary. Let $\mathcal{C}$ be a semi-abelian category. Recall that we classically define two functors

$$
\begin{aligned}
\pi_{0}: \operatorname{Gpd}(\mathcal{C}) \rightarrow \mathcal{C}, & \underline{G} \mapsto \operatorname{Coeq}\left(d_{\underline{G}}, c_{\underline{G}}\right) \\
\pi_{1}: \operatorname{Gpd}(\mathcal{C}) \rightarrow \mathcal{C}, \quad \underline{G} & \mapsto \operatorname{Ker}\left(d_{\underline{G}}\right) \cap \operatorname{Ker}\left(c_{\underline{G}}\right)
\end{aligned}
$$

It is immediate to state the following characterization:

Corollary 6.4. An internal functor $\underline{f}: \underline{H} \rightarrow \underline{G}$ is final if and only if $\pi_{0}(\underline{f})$ is an isomorphism, and $\pi_{1}(\underline{f})$ is a regular epimorphism.

It is interesting to wonder whether this result has a counterpart in a non-pointed environment. This subject will be investigated in a forthcoming paper. 


\subsection{Factorization of morphisms of extensions}

Given a semi-abelian category $\mathcal{C}$, we consider the category $\operatorname{EXT}_{\mathcal{C}}$, whose objects are extensions

$$
0 \longrightarrow K \stackrel{i}{\longrightarrow} X \stackrel{f}{\longrightarrow} Y \longrightarrow 0
$$

and morphisms are the obvious triples of arrows. Now, $\mathrm{EXT}_{\mathcal{C}}$ can be seen as the (full) subcategory of $\operatorname{XMod}(\mathcal{C})$ with objects the normal monomorphisms, hence we can give an account on the two factorization systems described in the previous section, restricted to extensions.

Proposition 6.5. In the category $\mathrm{EXT}_{\mathcal{C}}$, final morphisms coincide with $\pi_{0^{-}}$ invertible ones, and discrete fibrations coincide with $\pi_{0}$-cartesian morphisms.

Proof. The proof easily follows from the constructions above and from the characterization of final morphisms given in Proposition 3.12.

Hereafter, for an arrow $y$ with codomain $Y$, we denote by $y^{*}((i, f))$ the short exact sequence given by a pullback of $f$ along $y$, inducing the identity between the kernels. Dually, for an arrow $k$ with domain $K$, we denote by $k_{*}((i, f))$, whenever it exists, the short exact sequence given by a push forward of $i$ along $k$, inducing the identity between the cokernels.

Corollary 6.6. Let us consider the following diagram of solid arrows:

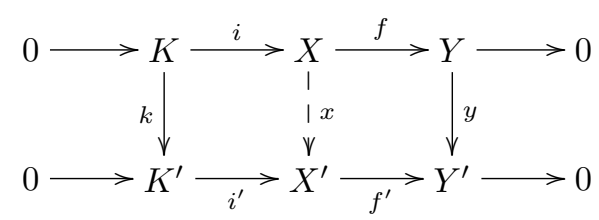

where the horizontal sequences are short exact. Then, there exists an arrow $x$ making the two squares commute if and only if $k_{*}((i, f))$ exists, and it is isomorphic to $y^{*}\left(\left(i^{\prime}, f^{\prime}\right)\right)$.

Proof. Let us suppose that such an $x$ exists. By Proposition 6.5 above, we get a factorization

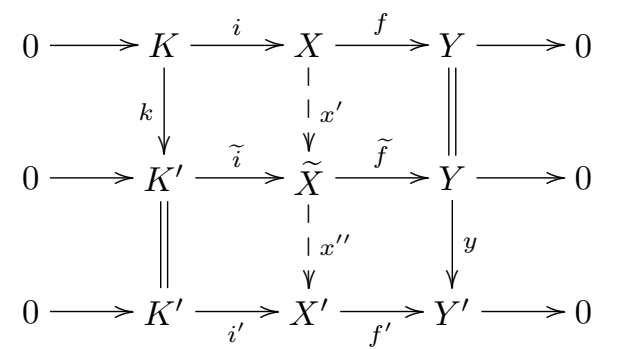

with $x^{\prime \prime} \cdot x^{\prime}=x$. By Proposition 3.12, the upper morphism of extensions is a push forward, while the right lower square is obviously a pullback.

The converse is trivial. 
This issue can be described somehow more explicitly when dealing with abelian extensions.

Corollary 6.7. Let us consider the following diagram of solid arrows:

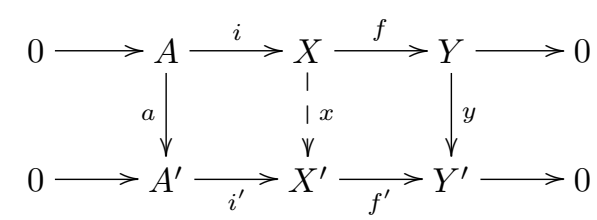

where the horizontal extensions are abelian, and let

$$
\xi: Y b A \rightarrow A, \quad \xi^{\prime}: Y^{\prime} b A^{\prime} \rightarrow A^{\prime}
$$

be the induced actions. Then, there exists an arrow $x$ making the two squares commute if and only if the pair $(a, y)$ induces a morphisms of modules and $a_{*}((i, f)) \cong y^{*}\left(\left(i^{\prime}, f^{\prime}\right)\right)$.

Proof. The extension $a_{*}((i, f))$ exists since $a$ obviously induces a morphism of $Y$-modules between $(A, \xi)$ and $\left(A^{\prime}, y^{*}\left(\xi^{\prime}\right)\right)$.

\section{Acknowledgments}

The first author was partially supported by FSE, Regione Lombardia.

\section{References}

[1] J. M. Beck, Triples, algebra and cohomology, 1967. Available in Reprints in Theory and Applications of Categories, 2 (2003) 1-59.

[2] F. Borceux And D. Bourn, Mal'cev, Protomodular, Homological and Semi-abelian Categories, Kluwer Academic Publishers (2004).

[3] D. Bourn, The shift functor and the comprehensive factorization for internal groupoids, Cahiers de Topologie et Géométrie Différentielle Catégoriques 28 (1987) 197-226.

[4] D. Bourn, Aspherical abelian groupoids and their directions, Journal of Pure and Applied Algebra 168 (2002) 133-146.

[5] D. BouRn, Internal profunctors and commutator theory; applications to extensions classification and categorical Galois Theory, Theory and Applications of Categories 24 (2010) 451-488.

[6] D. Bourn And G. Janelidze, Protomodularity, descent and semi-direct products, Theory and Applications of Categories 4 (1998) 37-46. 
[7] D. Bourn And G. JAnelidze, Extensions with abelian kernel in protomodular categories, Georgian Mathematical Journal 11 (2004) 645-654.

[8] D. Bourn And G. Janelidze, Centralizers in action accessible categories, Cahiers de Topologie et Géométrie Différentielle Catégoriques 50 (2009) 211232.

[9] D. Bourn And D. Rodelo, Comprehensive factorization and universal $I$ central extensions in the Mal'cev context, J. Pure Appl. Algebra 216 (2012) 598617 .

[10] K. S. Brown, Cohomology of groups, Springer-Verlag, (1982).

[11] M. Hartu, Push forward of crossed modules, abstract presented at Workshop on Category Theory, Coimbra 2012, July 2012.

[12] G. JAnelidze, Internal crossed modules, Georgian Mathematical Journal 10 (2003) 99-114.

[13] J. M. Casas, T. Datuashvili, M. Ladra, Universal strict general actors and actors in categories of interest, Appl. Categ. Structures 18 (2010) 85114.

[14] J.-L. Loday, Une version non commutative des algèbres de Lie: les algèbres de Leibniz, Enseign. Math 39 (1993) 269-293.

[15] S. Mac Lane, Homology, Springer-Verlag, (1963).

[16] S. Mantovani And G. Metere, Internal crossed modules and Peiffer condition, Theory and Applications of Categories 23 (2010) 113-135.

[17] S. Mantovani and G. Metere, Normalities and commutators, J. Algebra 324 (2010) 2568-2588.

[18] S. Mantovani, G. Metere and E. M. Vitale, Profunctors in Mal'tsev categories and fractions of functors, J. Pure Appl. Algebra (2013) in press.

[19] N. Martins-Ferreira And T. VAn Der Linden, A note on the "Smith is Huq" condition, Applied Categorical Structures 20 (2012) 175-187.

[20] B. NooHi, On weak maps between 2-groups, arXiv:math/0506313v3.

[21] G. Orzech, Obstruction theory in algebraic categories, I, J. Pure and Appl. Algebra 2 (1972), 287-314.

[22] P. T. Johnstone, Topos theory, Academic Press, London (1977).

[23] R. Street, R. F. C. Walters, The comprehensive factorization of a functor, Bull. Amer. Math. Soc. 79 (1973) 936-941. 